\title{
Impact loading during distracted running before and after auditory gait retraining
}

\begin{tabular}{|c|c|}
\hline Journal: & International Journal of Sports Medicine \\
\hline Manuscript ID & IJSM-04-2018-6920-ob.R2 \\
\hline Manuscript Type: & Orthopedics \& Biomechanics \\
\hline Key word: & Wearable sensor, Accelerometer, Vertical loading rate, Dual task, Kinetics \\
\hline Abstract: & $\begin{array}{l}\text { Visual feedback gait retraining has been reported to successfully reduce } \\
\text { impact loading in runners, even when the runners were distracted. } \\
\text { However, auditory feedback is more feasible in real life application. Hence, } \\
\text { this study compared the peak positive acceleration (PPA), vertical average } \\
\text { (VALR) and instantaneous (VILR) loading rate during distracted running } \\
\text { before and after a course of auditory feedback gait retraining in } 16 \\
\text { runners. They were asked to land with softer footfalls with and without } \\
\text { auditory feedback. Low or high sound pitch was generated according to the } \\
\text { impact of particular footfall, when compared with the preset target. } \\
\text { Runners then received a course of auditory gait retraining program and } \\
\text { after the gait retraining, runners completed a reassessment. Runners } \\
\text { before gait retraining exhibited lower PPA, VALR, and VILR with augmented } \\
\text { auditory feedback ( } p<0.049 \text { ). We found a reduction in PPA, VALR, and } \\
\text { VILR after gait retraining, regardless of the presence of feedback } \\
\text { ( } p<0.018 \text { ). However, runners after gait retraining did not demonstrate } \\
\text { further reduction in PPA and VALR with auditory feedback ( } p>0.104 \text { ). A } \\
\text { trivial effect of auditory feedback on VILR in runners after gait retraining } \\
\text { was observed ( } p=0.032 \text { ). Real time auditory feedback gait retraining is } \\
\text { effective in impact loading reduction, even when the runners were } \\
\text { distracted. }\end{array}$ \\
\hline
\end{tabular}

\section{SCHOLARONE ${ }^{m}$ \\ Manuscripts}




\title{
Impact loading during distracted running before and after auditory gait retraining
}

\begin{abstract}
Visual feedback gait retraining has been reported to successfully reduce impact loading in runners, even when the runners were distracted. However, auditory feedback is more feasible in real life application. Hence, this study compared the peak positive acceleration (PPA), vertical average (VALR) and instantaneous (VILR) loading rate during distracted running before and after a course of auditory feedback gait retraining in 16 runners. They were asked to land with softer footfalls with and without auditory feedback. Low or high sound pitch was generated according to the impact of particular footfall, when compared with the preset target. Runners then received a course of auditory gait retraining program and after the gait retraining, runners completed a reassessment. Runners before gait retraining exhibited lower PPA, VALR, and VILR with augmented auditory feedback $(p<0.049)$. We found a reduction in PPA, VALR, and VILR after gait retraining, regardless of the presence of feedback $(p<0.018)$. However, runners after gait retraining did not demonstrate further reduction in PPA and VALR with auditory feedback $(p>0.104)$. A small effect of auditory feedback on VILR in runners after gait retraining was observed $(p=0.032)$. Real time auditory feedback gait retraining is effective in impact loading reduction, even when the runners were distracted.
\end{abstract}

Keywords: Wearable sensor; Accelerometer; Vertical loading rate; Dual task; Kinetics 


\section{Introduction}

Impact loading parameters, such as vertical average loading rate (VALR), vertical instantaneous loading rate (VILR), and peak positive acceleration (PPA), have been associated with the development of overuse musculoskeletal injuries in distance runners $[11,35]$. A series of studies have reported successful impact loading reduction by real time biofeedback gait retraining $[8,9,31,32,34]$. More importantly, a recent large scale randomized controlled trial suggests such gait retraining may reduce injury risk in novice runners by $62 \%[6]$.

Among different running gait retraining protocols, vision is the most common tool used to convey feedback information [1], which is possibly due to a more convenient laboratory setup (i.e., participants looking at a screen placed in front of a treadmill). However, running with visual feedback in real life is not very practical. Therefore, previous studies have adopted auditory feedback such that participants adjust the running gait according to verbal instructions [13] and different sound pitches [34]. With the advancement of wearable sensors and smart garments, along with the fact that many runners listen to music during training, auditory feedback may allow a more feasible gait retraining outside laboratory environment.

Most of the current evidence in gait retraining was based on tests when the participants were only focused on the running task [11]. Since gait is not completely autonomous [12] and acquiring a new motor pattern after gait retraining may require certain level of attentional capacity, a recent study has examined the effectiveness of visual biofeedback gait retraining by challenging the participants with an additional 
cognitive task [7]. Interestingly, they found beneficial influences of the augmented visual feedback not only before, but also after gait retraining during dual-tasking. As participants after gait retraining may still rely on external cues for performing optimal motor pattern, such findings provide an argument that current visual gait retraining program may not be sufficient to lead to a stable modified gait pattern.

There is considerable evidence that human sensitivity and attention to purely temporal information is greater in the auditory than in the visual modality [14,16$20,23,26]$. Previous experiments have demonstrated that temporal discrimination and reproduction of temporal patterns are poorer in vision than in audition [17-20]. When auditory and visual stimuli are in conflict with respect to other modality at one of various phase relationships, participants' judgments are typically more strongly influenced by the auditory than by the visual temporal information $[14,16,23,26]$. Therefore, auditory feedback may be a better modality for gait retraining.

Hence, a replication of study by Cheung et al. [7] but using auditory feedback may provide insight on the level of motor learning using different sensory modality in gait retraining. Specifically, this study sought to compare the impact loading during distracted running before and after a course of auditory feedback gait retraining. Based on the previous findings [7], we hypothesized that participants would present a lower impact loading, as measured by PPA at shoe $\left(\mathrm{PPA}_{S}\right)$, PPA at distal tibia $\left(\mathrm{PPA}_{T}\right), \mathrm{VALR}$ and VILR, at the completion of gait retraining, when they were distracted. 


\section{Materials \& Methods}

\section{Study design}

The overall design of the present study referred to Cheung et al. [7]. Specifically, it is a cross-over study involving a laboratory-based gait retraining using auditory feedback. Participants were tested before and after gait retraining in two conditions i.e. with and without auditory feedback. This study meets the ethical standards outlined by the International Journal of Sports Medicine [22].

\section{Participants}

Sixteen recreational runners ( 9 females and 7 males; age $=25.1 \pm 7.9$ years; body height=1.64 $\pm 0.10 \mathrm{~m}$; body mass=57.37 $\pm 9.21 \mathrm{~kg}$; running experience $=3.2 \pm 0.9$ years; weekly mileage $=16.0 \pm 1.7 \mathrm{~km}$ ) who have been running for at least $12 \mathrm{~km}$ per week for a minimum of 12 months were recruited from local running clubs. They were free from any active injury twelve months before the experiment and had received no prior lower extremity surgery. In order to avoid floor effect, participants exhibited $\mathrm{PPA}_{S}<8 \mathrm{~g}$ during self-paced running were excluded [7]. Experimental procedures were reviewed and approved by the concerning institutional review board. Written consent was obtained from each participant before being tested. 


\section{Experimental procedures}

A lightweight tri-axial accelerometer (measurement range $=0-50 \mathrm{~g}$, Maestro WB, 01DB-Stell, Limonest, France) was aligned with the long axis of the participant's tibia, and securely affixed onto the posterior aspect of the right shoe. Another identical device was placed at the anteromedial right tibia [9] (Figure 1). At first, participants were asked to run at a self-selected pace on an instrumented treadmill (AMTI force sensing tandem treadmill, Watertown, MA, USA) for five minutes with their usual shoes. The running speed was kept constant within each participant throughout all of the tests. We set the target for gait retraining according to a previous study [7]. Specifically, participants were asked to soften their footfalls such that the PPAs was below $80 \%$ of the mean $\mathrm{PPA}_{S}$ from the last minute of the baseline assessment.

After establishing the target for gait retraining, participants were asked to land softer with and without auditory feedback in a randomized sequence generated by an online program (www.random.org). Simultaneously, they were required to conduct a cognitive and verbal counting task (i.e., addition of two to a random 3-digit integer) continuously for the entire 5-minute running trial [7]. Real time auditory feedback was given using a pair of stereo speakers located in front of the treadmill. Using customized LabVIEW code (National Instruments, TX, USA), a middle C $(261.6 \mathrm{~Hz})$ tone and a high-pitch C $(4,186.0 \mathrm{~Hz})$ of equal sound intensity were generated at a footfall below and above the preset loading target respectively. Participants were asked to modify their running gait in order to avoid the high-pitched sound. Running kinetics was measured during the last minute of the five-minute running bout in each condition [28]. A ten-minute rest was given between two trials in order to avoid fatigue. 
Participants then received a two-week gait retraining for impact loading reduction according to the protocol employed in Cheung et al. [7]. This gait retraining program was also employed by other previous studies $[3,6,9,25,33]$. In brief, they participated in eight sessions of gait modification over two weeks (four sessions per week). During the gait retraining, participants were asked to run with softer footfalls at the previous test speed on a treadmill. Similar to previous studies [6], participants were allowed to use their preferred strategies to achieve the goal. The same target and auditory feedback were used in the gait retraining. The gait retraining time was gradually increased from 15 minutes to 30 minutes over the eight sessions and auditory feedback was progressively removed in the last four sessions [9]. No verbal counting task was required during the gait retraining program. After the gait retraining, participants returned to complete a post-training assessment, which was identical to the pre-training assessment. Additionally, we asked if the participants experienced any symptoms or any other adverse effect related to the gait retraining. The overall study design is presented in Figure 2.

\section{Data acquisition and processing}

The accelerometer data was sampled at $1,000 \mathrm{~Hz}$ and filtered using a second order Butterworth low-pass filter at $50 \mathrm{~Hz}$. $\mathrm{PPA}_{S}$ and $P P A_{T}$ were defined as the maximum positive vertical acceleration that measured from the shoe-mounted sensor and the tibial sensor within the first $50 \mathrm{~ms}$ of foot-ground contact [10]. Synchronized vertical ground reaction force was recorded at $1,500 \mathrm{~Hz}$. Ground reaction force data was filtered using a fourth order Butterworth low-pass filter at $100 \mathrm{~Hz}$ and body weight normalized. 
The VALR and VILR were calculated as per the method described previously [2]. In brief, VALR is the slope of the line from the $20 \%$ point to the $80 \%$ point of the impact peak. VILR is the maximum slope of the vertical ground reaction force curve between the successive data points in the same region. In case of a non-clearly discernible impact peak, a set time point of $13 \%$ stance was used as a surrogate measure of the impact peak [4]. All the data were extracted and averaged across all the footfalls with the final one-minute of the trials.

Statistical analysis

A two-way repeated measures ANOVA was used to measure within-subject difference of PPA,$P P A_{T}$, VALR, and VILR between conditions (pre- and post-training; with and without feedback) during distracted running. All statistical tests were performed by SPSS software (Version 23, SPSS Inc., Chicago, IL, USA), with global alpha at 0.05 . In addition, differences between conditions were also illustrated by Cohen's d, where values greater than $0.2,0.5$ and 0.8 are considered as 'small, 'medium' and 'large' effect sizes respectively [30].

\section{Results}

All the participants completed gait retraining without any adverse effect reported. The self-selected running speed was $2.08 \pm 0.23 \mathrm{~m} / \mathrm{s}$. The PPA $, P P A_{T}, V A L R$, and VILR before and after gait retraining are presented in Table 1. There was only significant interaction effect between gait retraining and feedback on $P P A_{S}(F=16.129$; 
$p=0.001)$, while the interaction effects on $\mathrm{PPA}_{T}(\mathrm{~F}=1.428 ; p=0.266)$, VALR $(\mathrm{F}=3.438$; $p=0.083)$, and VILR $(\mathrm{F}=2.799 ; p=0.115)$ were not significant.

Participants presented a reduction in PPA $, P P A_{T}, V A L R$, and VILR after gait retraining, regardless of the presence of feedback $(p<0.018$; Cohen's $d=0.42-1.23$; moderate to large effect; see Table 2). Runners before gait retraining exhibited lower $\mathrm{PPA}_{S}, \mathrm{PPA}_{\mathrm{T}}, \mathrm{VALR}$, and VILR with augmented auditory feedback $(p<0.049$; Cohen's $d=0.27-0.55$; moderate to large effect; see Table 3 ). However, runners after gait retraining did not demonstrate further reduction in PPA, PPA $A_{T}$, and VALR with auditory feedback ( $p>0.104)$. We observed a small effect of auditory feedback on VILR in runners after gait retraining ( $p=0.032$; Cohen's $d=0.15$; Table 3$)$.

\section{Discussion}

The present study evaluated the effect of auditory feedback gait retraining on impact loading reduction in runners with cognitive distraction. We found that auditory gait retraining is effective in impact loading reduction. In addition, we found that augmented auditory feedback only reduces impact loading before gait retraining. Runners after gait retraining do not exhibit a difference in impact loading with additional feedback.

In the present study, we employed a cognitive and verbal counting task to distract the participants and better reflect the motor learning outcome after gait retraining. Such dual-task paradigm has been applied in some previous gait retraining studies $[5,7,27]$. According to the learning theory by Fitts and Posner [15], participants after the gait retraining should be able to perform the newly acquired gait pattern with less cognitive 
demand. Therefore, it is logical to test the effectiveness of gait retraining when the participants were distracted.

Except for the type of biofeedback adopted for gait retraining, our study was highly comparable with the work by Cheung et al. [7]. Cheung et al. reported that an augmented visual biofeedback after gait retraining may still be effective in lowering impact loading. In contrast, our study indicated that after same duration and intensity of gait retraining using auditory feedback, trained runners did not present further impact loading reduction with additional biofeedback. According to the learning theory by Fitts and Posner [15], there are three motor learning stages, namely cognitive stage, associative stage, and autonomous stage. It has been shown that augmented feedback are highly effective in either cognitive or associative stage [29]. Once the new gait pattern is mastered and autonomously performed, additional feedback should not affect performance. Given our study and the study by Cheung et al. [7] adopted identical duration and intensity of gait retraining protocol, our findings may potentially indicate a better motor learning from gait retraining with auditory feedback than visual feedback.

Previous studies have reported successful reduction in impact loading with moderate to large effect by a course of visual feedback gait retraining (Cohen's $d$ ranging between 0.74 and 1.7) [17-20]. Such effect is highly comparable with our findings. Our study is also in accordance with the findings reported by previous auditory gait retraining study [34], which indicates that auditory feedback gait retraining is effective in reducing impact loading in distance runners. However, some previous studies $[8,9]$ reported greater effect (Cohen's $d$ up to 1.7) than our findings, which can be explained by the difference in the gait retraining target. Our target was set at $80 \%$ of the original PPAs, while the 
other studies used $50 \%$ as the target for gait retraining $[8,9]$. Another possible reason may be due to the feedback used in the gait retraining. One of the previous studies used step rate as a feedback [21] and cadence can be measured by runners outside the laboratory environment. Additional practice may provide further improvement in gait modification and thus a greater effect was observed.

Previous experiments have demonstrated that the differentiation and reproduction of temporal patterns are stronger in audition than vision [17-20], suggesting auditory sensitivity and attention is greater than that in the visual modality. Some studies also reported participants' determinations are highly influenced by the auditory than by the visual temporal information $[14,16,23]$. Hence, auditory feedback may be a better modality for gait retraining than visual feedback. In real life, runners may modify their running mechanics with auditory signals, without losing focus on road conditions.

The results reported in this study may indicate a preference on the feedback type employed in gait retraining. On top of more convenient user interface, our findings may advocate the development of in-field auditory gait retraining with wearable sensors. Infield gait retraining programs using wearables has been reported as effective in modifying faulty gait biomechanics, such as lowering knee joint contact force during running [24,32]. This would allow a longer and more persistent gait retraining in the community, which may lead to a more persistent change in the motor performance during gait.

When interpreting our results, it is important to consider several limitations in our study. Firstly, only healthy recreational runners were recruited and hence our findings may not be generalized to runners at different elite levels or injured runners. Secondly, 
we affixed the accelerometer at the heel counter, as it is a common placement site for commercial biosensors. However, we did not measure participants' footstrike patterns, which may affect PPA measurement. Besides, some commercial sensors are mounted onto the lace box and may produce different results to this study. Future study is warranted to replicate the present experiment with different sensor placement locations. Thirdly, we did not measure the effect of gait retraining after each session. Therefore, the learning curve during the gait retraining program remains unknown. Finally, we did not measure joint kinematics. Since the participants may use different strategy to soften the footfalls, further study could explore the relationship between the modified kinetics and the running kinematics after gait retraining in future studies.

In conclusion, real time auditory feedback gait retraining is effective in impact loading reduction during distracted running. Runners after gait training do not benefit from augmented auditory feedback. When compared with findings reported by a visual feedback gait retraining study with similar design, auditory feedback gait retraining may provide better motor learning than gait retraining with visual feedback.

\author{
Acknowledgements \\ $X X X$
}




\section{References}

1 Agresta C, Brown A. Gait Retraining for Injured and Healthy Runners Using Augmented Feedback: A Systematic Literature Review. J Orthop Sports Phys Ther 2015; 45: 576-584

2 An W, Rainbow MJ, Cheung RTH. Effects of Surface Inclination on the Vertical Loading Rates and Landing Pattern during the First Attempt of Barefoot Running in Habitual Shod Runners. BioMed Res Int 2015; 2015: 240153

3 Barrios JA, Crossley KM, Davis IS. Gait retraining to reduce the knee adduction moment through real-time visual feedback of dynamic knee alignment. J Biomech 2010; 43: 2208-2213

4 Blackmore T, Willy RW, Creaby MW. The high frequency component of the vertical ground reaction force is a valid surrogate measure of the impact peak. J Biomech 2016; 49: 479-483

5 Caldwell LK, Laubach LL, Barrios JA. Effect of specific gait modifications on medial knee loading, metabolic cost and perception of task difficulty. Clin Biomech 2013; 28: 649-654

6 Chan ZYS, Zhang JH, Au IPH, An WW, Shum GLK, Ng GYF, Cheung RTH. Gait Retraining for the Reduction of Injury Occurrence in Novice Distance Runners: 1Year Follow-up of a Randomized Controlled Trial. Am J Sports Med 2017; 363546517736277

7 Cheung RTH, An WW, Au IPH, Zhang JH, Chan ZYS, MacPhail AJ. Control of impact loading during distracted running before and after gait retraining in runners. J Sports Sci $2017 ; 1-5$

8 Clansey AC, Hanlon M, Wallace ES, Nevill A, Lake MJ. Influence of tibial shock feedback training on impact loading and running economy. Med Sci Sports Exerc 2014; 46: 973-981

9 Crowell HP, Davis IS. Gait retraining to reduce lower extremity loading in runners. Clin Biomech Bristol Avon 2011; 26: 78-83

${ }^{10}$ Crowell HP, Milner CE, Hamill J, Davis IS. Reducing Impact Loading During Running With the Use of Real-Time Visual Feedback. J Orthop Sports Phys Ther 2010; 40: 206-213

${ }^{11}$ Davis IS, Futrell E. Gait Retraining: Altering the Fingerprint of Gait. Phys Med Rehabil Clin N Am 2016; 27: 339-355

${ }^{12}$ Dubost V, Annweiler C, Aminian K, Najafi B, Herrmann FR, Beauchet O. Stride-tostride variability while enumerating animal names among healthy young adults: result 
of stride velocity or effect of attention-demanding task? Gait Posture 2008; 27 : 138143

${ }^{13}$ Eriksson M, Halvorsen KA, Gullstrand L. Immediate effect of visual and auditory feedback to control the running mechanics of well-trained athletes. J Sports Sci 2011; 29: 253-262

${ }^{14}$ Fendrich $R$, Corballis PM. The temporal cross-capture of audition and vision. Percept Psychophys 2001; 63: 719-725

${ }^{15}$ Fitts PM, Posner MI. Human Performance. Oxford, England: Brooks/Cole Publishing Company, 1967

${ }^{16}$ Goldstone S, Boardman WK, Lhamon WT. Intersensory comparisons of temporal judgments. J Exp Psychol 1959; 57: 243-248

17 Goodfellow LD. An Empirical Comparison of Audition, Vision, and Touch in the Discrimination of Short Intervals of Time. Am J Psychol 1934; 46: 243

${ }^{18}$ Grondin S. Duration discrimination of empty and filled intervals marked by auditory and visual signals. Percept Psychophys 1993; 54: 383-394

${ }^{19}$ Grondin S, Meilleur-Wells G, Ouellette C, Macar F. Sensory effects on judgments of short time-intervals. Psychol Res 1998; 61: 261-268

${ }^{20}$ Grondin S, Ouellet B, Roussel M-\&Egrave;ve. About optimal timing and stability of Weber fraction for duration discrimination. Acoust Sci Technol 2001; 22: 370-372

${ }^{21}$ Hafer JF, Brown AM, deMille P, Hillstrom HJ, Garber CE. The effect of a cadence retraining protocol on running biomechanics and efficiency: a pilot study. J Sports Sci 2015; 33: 724-731

${ }^{22}$ Harriss D, Macsween A, Atkinson G. Standards for Ethics in Sport and Exercise Science Research: 2018 Update. Int J Sports Med 2017; 38: 1126-1131

${ }^{23}$ Morein-Zamir S, Soto-Faraco S, Kingstone A. Auditory capture of vision: examining temporal ventriloquism. Brain Res Cogn Brain Res 2003; 17: 154-163

${ }^{24}$ Napier C, Esculier J-F, Hunt MA. Gait retraining: out of the lab and onto the streets with the benefit of wearables. Br J Sports Med 2017; 51: 1642-1643

${ }^{25}$ Noehren B, Scholz J, Davis I. The effect of real-time gait retraining on hip kinematics, pain and function in subjects with patellofemoral pain syndrome. Br J Sports Med 2011; 45: 691-696

${ }^{26}$ Recanzone $\mathrm{GH}$. Auditory influences on visual temporal rate perception. J Neurophysiol 2003; 89: 1078-1093 
${ }^{27}$ Richards R, van der Esch M, van den Noort JC, Harlaar J. The learning process of gait retraining using real-time feedback in patients with medial knee osteoarthritis. Gait Posture 2018; 62: 1-6

28 Riley PO, Dicharry J, Franz J, Croce UD, Wilder RP, Kerrigan DC. A Kinematics and Kinetic Comparison of Overground and Treadmill Running: Med Sci Sports Exerc 2008; 40: 1093-1100

${ }^{29}$ Schmidt R, Lee T. Motor Learning and Performance: from principles to application. 5th Edition. IL, USA: Human Kinetics, 2014

${ }^{30}$ Sullivan GM, Feinn R. Using Effect Size-or Why the P Value Is Not Enough. J Grad Med Educ 2012; 4: 279-282

31 Tate JJ, Milner CE. Sound intensity feedback during running reduces loading rates and impact peak. J Orthop Sports Phys Ther 2017; Epub July 6, 2017: 1-18

${ }^{32}$ Willy RW, Buchenic L, Rogacki K, Ackerman J, Schmidt A, Willson JD. In-field gait retraining and mobile monitoring to address running biomechanics associated with tibial stress fracture. Scand J Med Sci Sports 2015;

${ }^{33}$ Willy RW, Scholz JP, Davis IS. Mirror gait retraining for the treatment of patellofemoral pain in female runners. Clin Biomech 2012; 27: 1045-1051

${ }^{34}$ Wood CM, Kipp K. Use of audio biofeedback to reduce tibial impact accelerations during running. J Biomech 2014; 47: 1739-1741

${ }^{35}$ van der Worp $H$, Vrielink JW, Bredeweg SW. Do runners who suffer injuries have higher vertical ground reaction forces than those who remain injury-free? A systematic review and meta-analysis. Br J Sports Med 2016; 50: 450-457 


\section{Figure legends}

Figure 1. Sensor locations (indicated by the red boxes) and corresponding fixations

Figure 2. Study flow diagram

\section{Table legends}

Table 1. Peak positive acceleration measured at the shoe (PPA $)$, peak positive acceleration measured at the distal tibia $\left(P P A_{T}\right)$, vertical average loading rate (VALR) and vertical instantaneous loading rate (VILR) before and after gait retraining

Table 2. Differences of peak positive acceleration measured at the shoe (PPAs), peak positive acceleration measured at the distal tibia $\left(\mathrm{PPA}_{\mathrm{T}}\right)$, vertical average loading rate (VALR) and vertical instantaneous loading rate (VILR) before and after gait retraining Table 3. Effect of augmented auditory feedback on peak positive acceleration measured at the shoe $\left(\mathrm{PPA}_{S}\right)$, peak positive acceleration measured at the distal tibia $\left(\mathrm{PPA}_{T}\right)$, vertical average loading rate (VALR) and vertical instantaneous loading rate (VILR) 
Table 1. Peak positive acceleration measured at the shoe (PPA $)$, peak positive acceleration measured at the distal tibia (PPAT), average vertical loading rate (VALR) and instantaneous vertical loading rate (VILR) before and after gait retraining

\begin{tabular}{|c|c|c|c|c|}
\hline \multirow[b]{2}{*}{ Parameters } & \multicolumn{2}{|c|}{ Pre-training } & \multicolumn{2}{|c|}{ Post-training } \\
\hline & Without auditory feedback & With auditory feedback & Without auditory feedback & With auditory feedback \\
\hline $\operatorname{PPA}_{S}(g)$ & $19.01 \pm 7.71$ & $14.78 \pm 7.16$ & $12.58 \pm 6.91$ & $11.61 \pm 7.53$ \\
\hline $\mathrm{PPA}_{T}(\mathrm{~g})$ & $5.45 \pm 1.63$ & $4.91 \pm 1.37$ & $4.28 \pm 1.50$ & $3.92 \pm 1.32$ \\
\hline $\operatorname{VALR}(\mathrm{N} / \mathrm{kg} / \mathrm{s})$ & $62.66 \pm 18.78$ & $56.61 \pm 19.72$ & $47.11 \pm 15.21$ & $45.39 \pm 14.92$ \\
\hline VILR (N/kg/s) & $74.63 \pm 19.96$ & $68.99 \pm 20.09$ & $58.34 \pm 17.11$ & $55.72 \pm 17.12$ \\
\hline
\end{tabular}


Table 2. Differences of peak positive acceleration measured at the shoe (PPA $)$, peak positive acceleration measured at the distal tibia $\left(P P A_{T}\right)$, vertical average loading rate (VALR) and vertical instantaneous loading rate (VILR) before and after gait retraining

\begin{tabular}{lcc}
\hline \hline Parameters & Cohen's d & P value \\
\hline PPA $_{S}$ without auditory feedback & 0.85 & $<0.001^{*}$ \\
PPA $_{S}$ with auditory feedback & 0.42 & $0.004^{*}$ \\
PPA $_{T}$ without auditory feedback & 0.95 & $0.018^{*}$ \\
PPA $_{T}$ with auditory feedback & 1.23 & $0.012^{*}$ \\
VALR without auditory feedback & 0.88 & $<0.001^{*}$ \\
VALR with auditory feedback & 0.62 & $0.001^{*}$ \\
VILR without auditory feedback & 0.85 & $<0.001^{*}$ \\
VILR with auditory feedback & 0.69 & $0.002^{*}$ \\
\hline \hline
\end{tabular}

* indicates significant difference before and after gait retraining 
Table 3. Effect of augmented auditory feedback on peak positive acceleration measured at the shoe $\left(P P A_{S}\right)$, peak positive acceleration measured at the distal tibia $\left(P P A_{T}\right)$, vertical average loading rate (VALR) and vertical instantaneous loading rate $(\mathrm{VILR})$

\begin{tabular}{lcc}
\hline \hline Parameters & Cohen's $d$ & $P$ value \\
\hline $\mathrm{PPA}_{S}$ before gait retraining & 0.55 & $<0.001^{*}$ \\
$\mathrm{PPA}_{S}$ after gait retraining & - & 0.158 \\
$\mathrm{PPA}_{T}$ before gait retraining & 0.45 & $0.049^{*}$ \\
$\mathrm{PPA}_{T}$ after gait retraining & - & 0.104 \\
VALR before gait retraining & 0.30 & $0.024^{*}$ \\
VALR after gait retraining & - & 0.195 \\
VILR before gait retraining & 0.27 & $0.014^{*}$ \\
VILR after gait retraining & 0.15 & $0.032^{*}$ \\
\hline \hline
\end{tabular}

* indicates significant difference with and without auditory feedback 

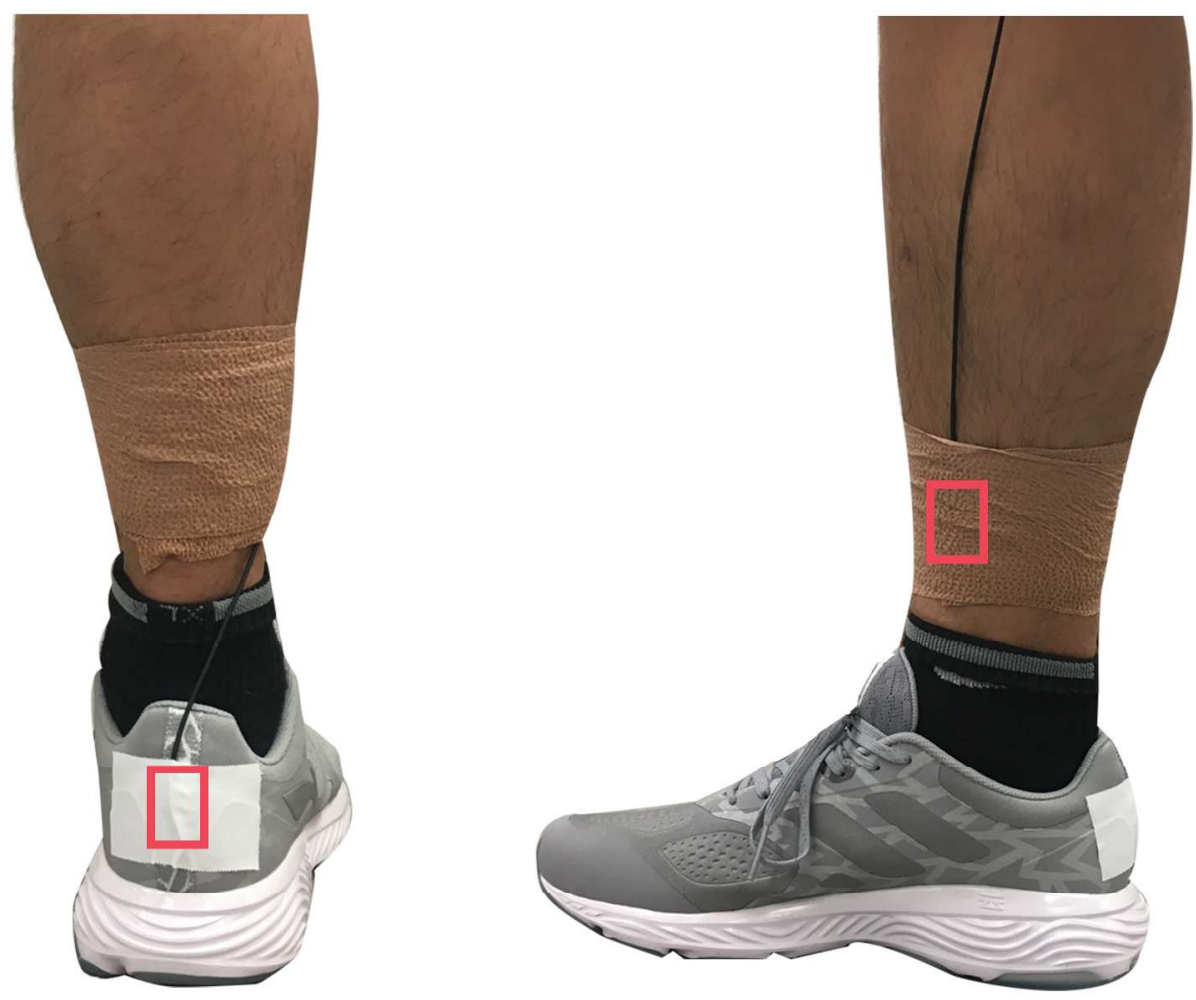

Fig. 1: Sensor locations (indicated by the red boxes) and corresponding fixations $438 \times 339 \mathrm{~mm}(300 \times 300 \mathrm{DPI})$ 


\section{Pre-training assessment ( $n=16)$}

With cognitive and verbal counting task

- Auditory feedback

- $\quad$ No feedback

(In a randomized sequence)

\section{Post-training assessment ( $\mathrm{n}=16)$}

With cognitive and verbal counting task

- Auditory feedback

- $\quad$ No feedback

(In a randomized sequence)

Fig. 2: Study flow diagram

$413 \times 669 \mathrm{~mm}(300 \times 300 \mathrm{DPI})$ 